\title{
Controllability of Singular Linear Systems by Legendre Wavelets
}

\author{
Wenxin Yu, ${ }^{1}$ Yigang $\mathrm{He}^{1,2}$ Xianming Wu, ${ }^{1}$ and $\mathrm{Kun} \mathrm{Gao}^{1}$ \\ ${ }^{1}$ College of Electrical and Information Engineering, Hunan University, Changsha, Hunan 410082, China \\ ${ }^{2}$ School of Electrical and Automation Engineering, Hefei University of Technology, Hefei, Anhui 230009, China
}

Correspondence should be addressed to Wenxin Yu; slowbird@sohu.com

Received 9 August 2014; Revised 26 October 2014; Accepted 28 October 2014; Published 20 November 2014

Academic Editor: Zengqiang Chen

Copyright (C) 2014 Wenxin Yu et al. This is an open access article distributed under the Creative Commons Attribution License, which permits unrestricted use, distribution, and reproduction in any medium, provided the original work is properly cited.

We propose a new method to design an observer and control the linear singular systems described by Legendre wavelets. The idea of the proposed approach is based on solving the generalized Sylvester equations. An example is also given to illustrate the procedure.

\section{Introduction}

Singular systems, also commonly called generalized or descriptor systems in the literature, appear in many practical situations including engineering systems, economic systems, network analysis, and biological systems. In fact, many systems in the real life are singular essentially. They are usually simplified or approximated by nonsingular models because there is still lacking of efficient tools to tackle problems related to such systems. The structural analysis of linear singular systems, using either algebraic or geometric approach, has attracted considerable attention from many researchers during the last three decades (see, e.g., [1-3]).

Since the introduction of Legendre wavelets method (LWM), for the resolution of variational problems, by Razzaghi and Yousefi in 2000 and 2001 [4, 5], several works applying this method were born. To mention a few, we give the resolution of differential equations [6], the study of optimal control problem with constraints [7], the resolution of linear integro-differential equations, the numerical resolution of Abel equation, and the resolution of fractional differential equations.

In this paper, we propose a new method to design an observer and control the linear singular systems described by Legendre wavelets. The method is based upon expanding various time functions in the system as their truncated Legendre wavelets. The operational matrix is introduced and utilized to reduce the solution of time singular linear system to the solution of algebraic equations. Finally, we obtain the interrelations between solution problems for the linear matrix equations of Sylvester with suitable controllability and observability conditions.

\section{Properties of Legendre Wavelets}

Wavelets are mathematical functions that are constructed using dilation and translation of a single function called the mother wavelet denoted by $\psi(t)$ and satisfied certain requirements.

If the dilation parameter is $a$ and translation parameter is $b$, then we have the following family of wavelets:

$$
\psi_{a, b}(t)=|a|^{1 / 2} \psi\left(\frac{t-b}{a}\right) \quad \text { with } a, b \in R, a \neq 0 \text {. }
$$

Restricting $a$ and $b$ to discrete values, such as $a=a_{0}^{-k}, b=$ $n b_{0} a_{0}^{-k}, a_{0}>1, b_{0}>0$, and $n, k$ are positive integers, we give

$$
\psi_{k, n}(t)=|a|^{k / 2} \psi\left(a_{0}^{k} t-n b_{0}\right)
$$

where $\psi_{k, n}(t)$ form a basis for $L^{2}(R)$. If $a_{0}=2$ and $b_{0}=1$, then it is clear that the set $\left\{\psi_{k, n}(t)\right\}$ forms an orthonormal basis for $L^{2}(R)$.

Legendre wavelets $\psi_{n, m}(t)=\psi(k, \widehat{n}, m, t)$ have four arguments: $\widehat{n}=2 n-1, n=1,2,3, \ldots, 2^{k-1}, k$ is assumed to be any positive integer, $m$ is the order for Legendre polynomials, 
and $t$ is the normalized time. They are defined on the interval $[0,1]$ as

$$
\psi_{n, m}=\left\{\begin{array}{cc}
\sqrt{m+\frac{1}{2}} 2^{k / 2} P_{m}\left(2^{k} t-\widehat{n}\right) & \text { for } \frac{\widehat{n}}{2^{k}} \leq t \leq \frac{\widehat{n}+1}{2^{k}} \\
0 & \text { otherwise }
\end{array}\right\}
$$

with $m=0,1, \ldots, M-1$ and $n=0,1, \ldots, 2^{k-1}$. The coefficient $\sqrt{m+1 / 2}$ is orthonormality. Here, $P_{m}(t)$ are the well-known Legendre polynomials of order $m$, which are defined on the interval $[-1,1]$ and can be determined with the aid of the following recurrence formulae $[8,9]$ :

$$
\begin{gathered}
P_{0}(t)=1, \quad P_{1}(t)=t, \\
P_{m+1}(t)=\left(\frac{2 m+1}{m+1}\right) t P_{m}(t)-\left(\frac{m}{m+1}\right) P_{m-1}(t), \\
m=1,2,3 \ldots
\end{gathered}
$$

A function $f(t)$ defined over $[0,1]$ can be expanded as

$$
\begin{aligned}
f(t) & =\sum_{n=0}^{\infty} \sum_{m \in Z} c_{n, m} \psi_{n, m}(t) \\
& \approx \sum_{n=0}^{2^{k}-1} \sum_{m=-M}^{M} c_{n, m} \psi_{n, m}(t)=C^{T} \psi(t),
\end{aligned}
$$

where

$$
c_{n, m}=\left(f(t), \psi_{n, m}(t)\right)=\int_{0}^{1} f(t) \psi_{n, m}(t) d t .
$$

$C$ and $\psi(t)$ are $2^{k}(2 M+1) \times 1$ vectors given by

$$
\begin{aligned}
& C=\left[c_{1,0}, c_{1,1}, \ldots, c_{1, M-1}, c_{2,0}, \ldots, c_{2,1}, \ldots,\right. \\
& \left.c_{2, M-1} c_{2^{k-1}, 0}, \ldots, c_{2^{k-1}, M-1}\right]^{T} \text {, } \\
& \psi(t)=\left[\psi_{1,0}, \psi_{1,1}, \ldots, \psi_{1, M-1}, \psi_{2,0}, \ldots, \psi_{2,0}, \ldots,\right. \\
& \left.\psi_{2^{K-1}, M-1}, \ldots, \psi_{2^{k-1}, M-1}\right]^{T} \text {. }
\end{aligned}
$$

The integration of the function $\psi(t)$ in $(5)$ is given by

$$
\int_{0}^{t} \psi(s) d s=P \psi(t),
$$

where $P$ is an $\left(2^{k-1} M\right) \times\left(2^{k-1} M\right)$ matrix, called the operational matrix, and is given by $[10,11]$

$$
P=\frac{1}{2^{k+1}}\left[\begin{array}{ccccc}
L & F & F & \cdots & F \\
0 & L & F & \cdots & F \\
\vdots & 0 & \ddots & \ddots & \vdots \\
0 & 0 & \cdots & 0 & L
\end{array}\right] \text {, }
$$

where $F$ and $L$ are $M \times M$ matrices given by

$$
F=\left[\begin{array}{cccc}
2 & 0 & \cdots & 0 \\
0 & 0 & \cdots & 0 \\
\vdots & \vdots & \vdots & \vdots \\
0 & 0 & \cdots & 0
\end{array}\right]
$$

L

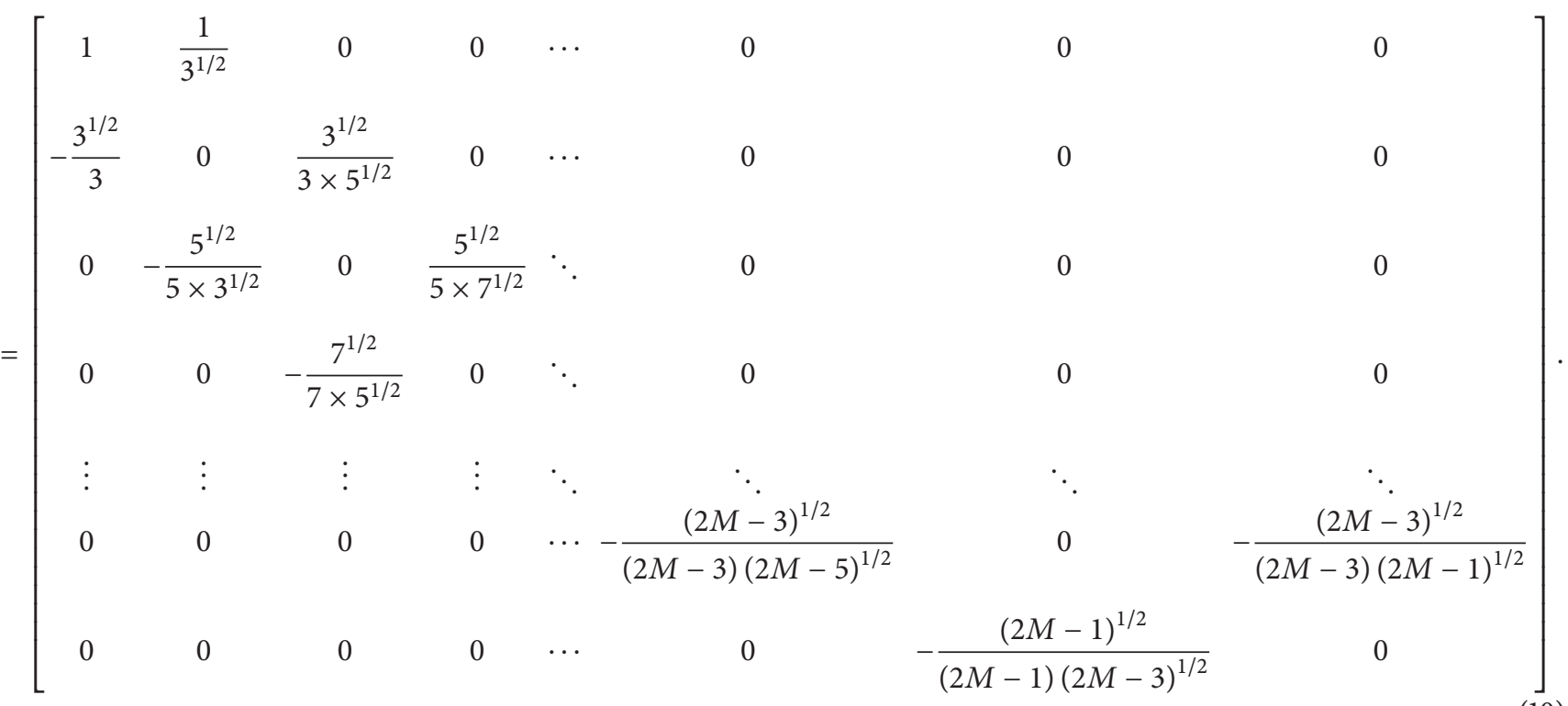


The integration of the product of two Legendre wavelet function vectors is obtained as

$$
\int_{0}^{1} \psi(t) \psi(t)^{T} d t=1
$$

\section{Properties of the Sylvester Equation and Model Described}

Consider the Sylvester equation

$$
A X-X B=\Phi_{1} \Phi_{2}
$$

where $A \in C^{n \times n}, B \in C^{m \times m}, X \in C^{n \times m}, \Phi_{1} \in C^{n \times k}$, and $\Phi_{2} \in C^{k \times m}$.

For some recent developments in the theory of rational matrix functions and in the theory of linear systems and control leading to equations, one can refer to [11-15].

Definition 1. Identify the least positive integer $k$ for which a solution $X, \Phi_{1}, \Phi_{2}$, with $\left(A, \Phi_{1}\right)$ is controllable and $\left(\Phi_{2}, B\right)$ is observable

$$
k(A, B)=\max _{\lambda \in C}\{\operatorname{dim} \operatorname{Ker}(\lambda I-A)+\operatorname{dim} \operatorname{Ker}(\lambda I-B)\} .
$$

Lemma 2. Let $A \in C^{n \times n}$ be given. There exists $\Phi_{1} \in$ $C^{n \times k}$ such that $\left(A, \Phi_{1}\right)$ is controllable, if and only if $k \geq$ $\max _{\lambda \in C} \operatorname{dimKer}(\lambda I-A)$.

Theorem 3. Given $A \in C^{n \times n}, B \in C^{m \times m}$. The minimal integer $K$ for which there exist $X \in C^{n \times m}, \Phi_{1} \in C^{n \times r}$, and $\Phi_{2} \in C^{r \times m}$ satisfying (12) such that $\left(A, \Phi_{1}\right)$ is controllable and $\left(\Phi_{2}, B\right)$ is observable, which is equal to $k(A, B)$.

A singular linear system can be described as follows:

$$
\begin{gathered}
K \dot{X}(t)=E X(t)+F U(t), \\
X(0)=X_{0},
\end{gathered}
$$

where $X \in R^{n}, U(t) \in R^{m}, K \in R^{n \times n}, E \in R^{n \times n}$, and $F \in$ $R^{n \bar{\mp} \times m}$.

\section{Solution of Singular Linear System}

In this section, the Legendre wavelet method is used for solving the singular linear system by approximated functions. Assume that $U(t)$ is square integral in the interval $[0,1)$. In the matrix forms,

$$
U(t)=G \psi(t)
$$

where $G$ is a $p \times m$ matrix. $G$ can be obtained by the method described in Section 2.

Avoiding impulse functions, we straight away expand $\dot{X}(t)$ instead of $X(t)$ itself into Legendre wavelet given by $\dot{X}(t)=S \psi(t)$. So we get

$$
X(t)=\int_{0}^{t} \dot{X}(\tau) d \tau=S \int_{0}^{t} \psi(\tau) d \tau+X_{0}=\operatorname{SP} \psi(t)+X_{0}
$$

By substituting (16) and (17) into (14), we obtain

$$
K S \psi(t)=E\left[S P \psi(t)+X_{0}\right]+F G \psi(t) .
$$

We have

$$
[K S-E S P] \psi(t)=\{Q+F G\} \psi(t),
$$

where $Q \psi(t)=E X_{0}$

In this work (19) can be written as

$$
K S-E S P=W,
$$

where

$$
W=\{Q+B G\}
$$

Since the unknown matrix in (20), it can be solved using Kronecker product as

$$
S=\left[K \otimes I-E \otimes P^{T}\right]^{-1} W^{T},
$$

where

$$
S^{T}=\left[\begin{array}{c}
s_{0} \\
s_{1} \\
\vdots \\
s_{m-1}
\end{array}\right], \quad W^{T}=\left[\begin{array}{c}
w_{0} \\
w_{1} \\
\vdots \\
w_{m-1}
\end{array}\right]
$$

So $X(t)=S \psi(t)$.

\section{Some Theorems}

For $K S-E S P=W$, if $E^{-1} \in R^{n \times n}$, then $E^{-1} K S-S P=E^{-1} W$; let $\widetilde{A}=E^{-1} K, \widetilde{B}=P, \widetilde{\Phi}_{1}=E^{-1}$, and $\widetilde{\Phi}_{2}=W$; we obtain $\widetilde{A} S-S \widetilde{B}=\widetilde{\Phi}_{1} \widetilde{\Phi}_{2}$

Accordingly, we propose some results.

Lemma 4. Let $\widetilde{A} \in R^{n \times n}$ be given. There exists $\widetilde{\Phi}_{1} \in$ $R^{n \times k}$ such that $\left(\widetilde{A}, \widetilde{\Phi}_{1}\right)$ is controllable, if and only if $k \geq$ $\max _{\lambda \in C} \operatorname{dimKer}(\lambda I-\widetilde{A})$.

Theorem 5. Given $\widetilde{A} \in R^{n \times n}, \widetilde{B} \in R^{m \times m}$. The minimal integer $K$ for which there exist $S \in R^{n \times m}, \widetilde{\Phi}_{1} \in R^{n \times r}$, and $\widetilde{\Phi}_{2} \in R^{r \times m}$ satisfying (24) such that $\left(\widetilde{A}, \widetilde{\Phi}_{1}\right)$ is controllable and $\left(\widetilde{\Phi}_{2}, \widetilde{B}\right)$ is observable, which is equal to $k(\widetilde{A}, \widetilde{B})$.

\section{Example}

For Legendre Wavelets, let $M=3, k=2$; let us consider the first order linear singular system:

$$
\left[\begin{array}{llll}
1 & 0 & 0 & 0 \\
0 & 0 & 0 & 0 \\
0 & 0 & 1 & 0 \\
0 & 0 & 0 & 0
\end{array}\right] x^{\prime}(t)=\left[\begin{array}{cccc}
0 & 1 & 0 & 0 \\
1 & 1 & 0 & 0 \\
0 & 0 & 0 & 1 \\
0 & -1 & 1 & 1
\end{array}\right] x(t)+\left[\begin{array}{ll}
0 & 0 \\
1 & 0 \\
0 & 0 \\
0 & 1
\end{array}\right] u \text {. }
$$

Set

$$
x_{0}=\left[\begin{array}{c}
0 \\
-1 \\
1 \\
-2
\end{array}\right], \quad u=\left[\begin{array}{l}
1 \\
0
\end{array}\right]
$$


We solve (24) using the algorithm described in Section 5 for the case corresponds to Theorem 5, so we can get (24) that is controllable and observable.

\section{Conclusions}

By the analysis of the above, we find that the method proposed in the paper is efficient in tackling the singular linear systems. Furthermore, the method is also applied for solving the interrelations between solution problems for singular linear systems and the linear matrix equations of Sylvester with suitable controllability and observability conditions. The design example is good enough to illustrate our idea.

\section{Conflict of Interests}

The authors declare that there is no conflict of interests regarding the publication of this paper.

\section{Acknowledgments}

This work was supported by the National Natural Science Foundation for Distinguished Young Scholars of China (Grant no. 50925727), the National Natural Science Foundation of China (Grant no. 60876022), the National Defense Advanced Research Project Grant nos. C1120110004, 9140A27020211DZ5102, the Key Grant Project of Chinese Ministry of Education under Grant no. 313018, Anhui Provincial Science and Technology Foundation of China under Grant no. 1301022036, and Hunan Provincial Science and Technology Foundation of China under Grant nos. 2010J4 and 2011JK202.

\section{References}

[1] P. Misra, P. Van Dooren, and A. Varga, "Computation of structural invariants of generalized state-space systems," Automatica, vol. 30, no. 12, pp. 1921-1936, 1994.

[2] K. Murugesan and V. Balakumar, "Study on singular systems of index three via Runge-Kutta method of order-10," International Journal of Pure and Applied Mathematics, vol. 70, no. 5, pp. 723733, 2011.

[3] M. Essabre, J. Soulami, and E. Elyaagoubi, "Design of state observer for a class of non linear singular systems described by Takagi-Sugeno model," Contemporary Engineering Sciences, vol. 6, no. 1-4, pp. 99-109, 2013.

[4] M. Razzaghi and S. Yousefi, "Legendre wavelets direct method for variational problems," Mathematics and Computers in Simulation, vol. 53, no. 3, pp. 185-192, 2000.

[5] M. Razzaghi and S. Yousefi, “The Legendre wavelets operational matrix of integration," International Journal of Systems Science: Principles and Applications of Systems and Integration, vol. 32, no. 4, pp. 495-502, 2001.

[6] X.-Y. Zheng, X.-F. Yang, and Y. Wu, "Technique for solving differential equation by extended Legendre wavelets," in Proceedings of the International Conference on Wavelet Analysis and Pattern Recognition (ICWAPR '08), pp. 667-671, Hong Kong, August 2008.
[7] M. Razzaghi and S. Yousefi, "Legendre wavelets method for constrained optimal control problems," Mathematical Methods in the Applied Sciences, vol. 25, no. 7, pp. 529-539, 2002.

[8] M. Razzaghi and S. Yousefi, "Legendre wavelets direct method for variational problems," Mathematics and Computers in Simulation, vol. 53, no. 3, pp. 185-192, 2000.

[9] M. Razzaghi and S. Yousefi, "Legendre wavelets method for constrained optimal control problems," Mathematical Methods in the Applied Sciences, vol. 25, no. 7, pp. 529-539, 2002.

[10] F. Mohammadi and M. M. Hosseini, "A new Legendre wavelet operational matrix of derivative and its applications in solving the singular ordinary differential equations," Journal of the Franklin Institute: Engineering and Applied Mathematics, vol. 348, no. 8, pp. 1787-1796, 2011.

[11] M. Razzaghi and S. Yousefi, "Legendre wavelets method for the solution of nonlinear problems in the calculus of variations," Mathematical and Computer Modelling, vol. 34, no. 1-2, pp. 4554, 2001.

[12] N. Irfan and S. Kapoor, "Quick glance on different wavelets and their operational matrix properties: a review," International Journal of Research and Reviews in Applied Sciences, vol. 8, no. 1, 2011.

[13] E. de Souza and S. P. Bhattacharyya, "Controllability, observability and the solution of $A X-X B=C$," Linear Algebra and its Applications, vol. 39, pp. 167-188, 1981.

[14] L. Lerer and L. Rodman, "Sylvester and Lyapunov equations and some interpolation problems for rational matrix functions," Linear Algebra and Its Applications, vol. 185, pp. 83-117, 1993.

[15] B. Zhou and G.-R. Duan, "A new solution to the generalized Sylvester matrix equation $A V-E V F=B W$," Systems and Control Letters, vol. 55, no. 3, pp. 193-198, 2006. 

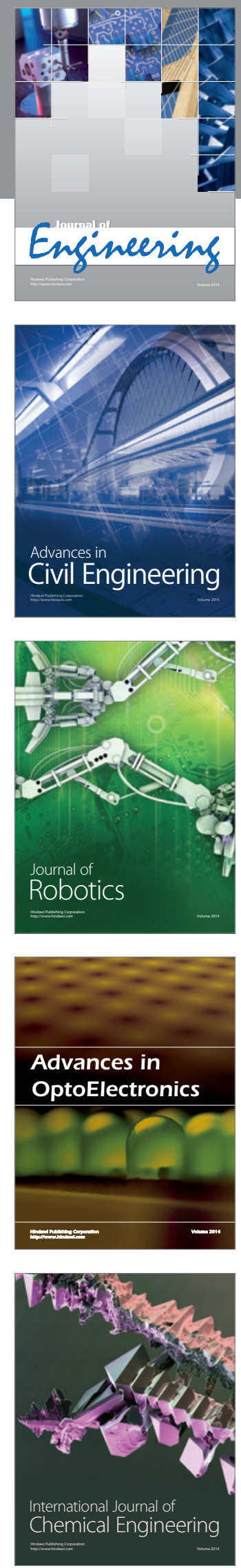

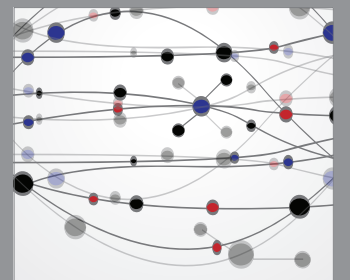

The Scientific World Journal
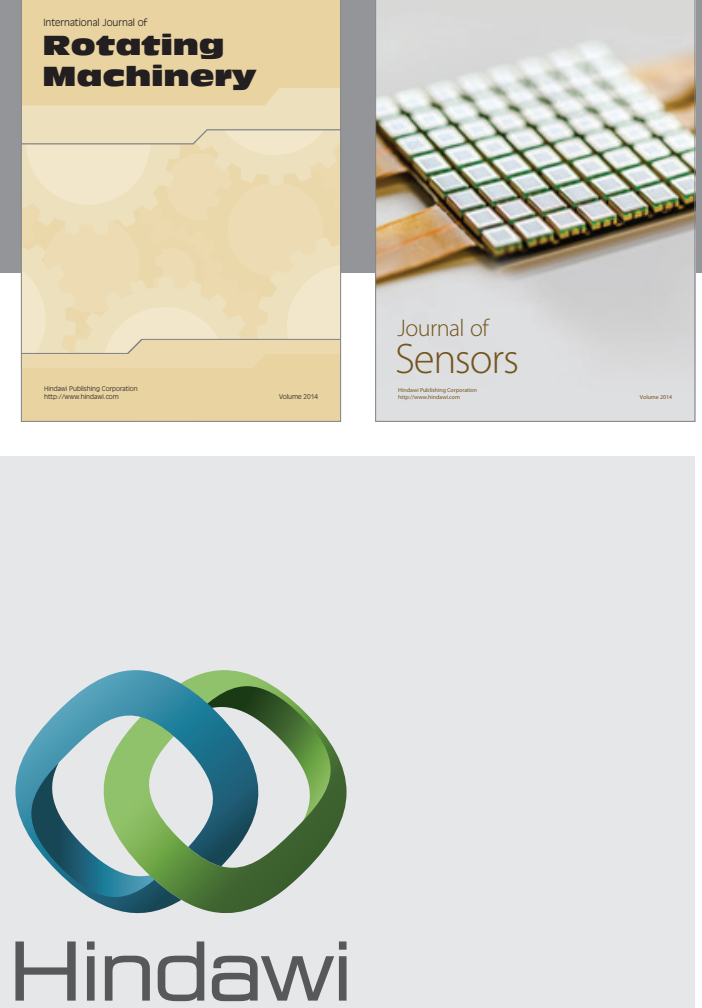

Submit your manuscripts at http://www.hindawi.com
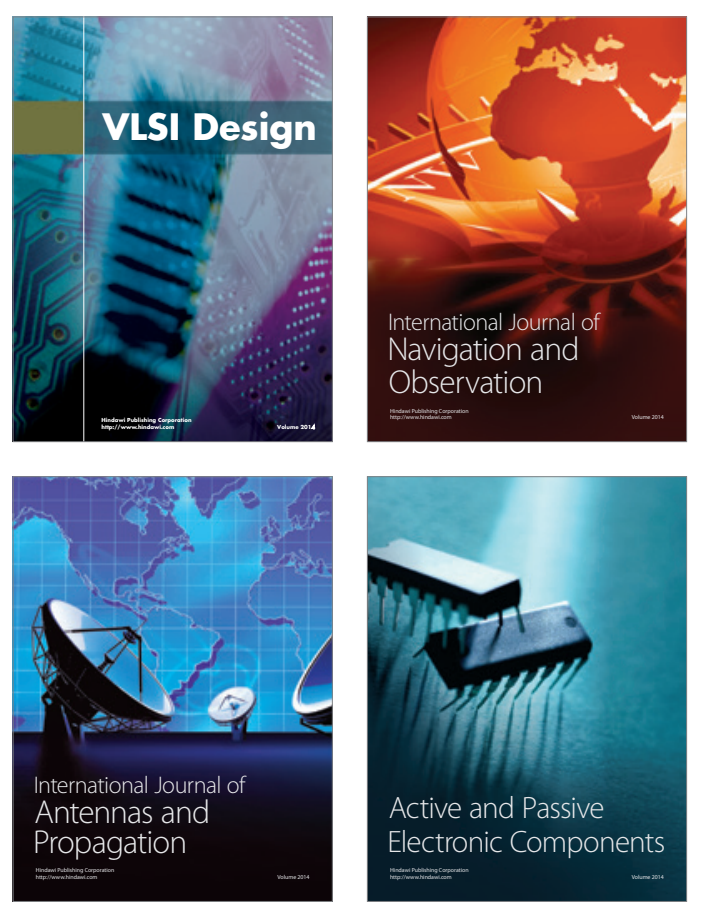
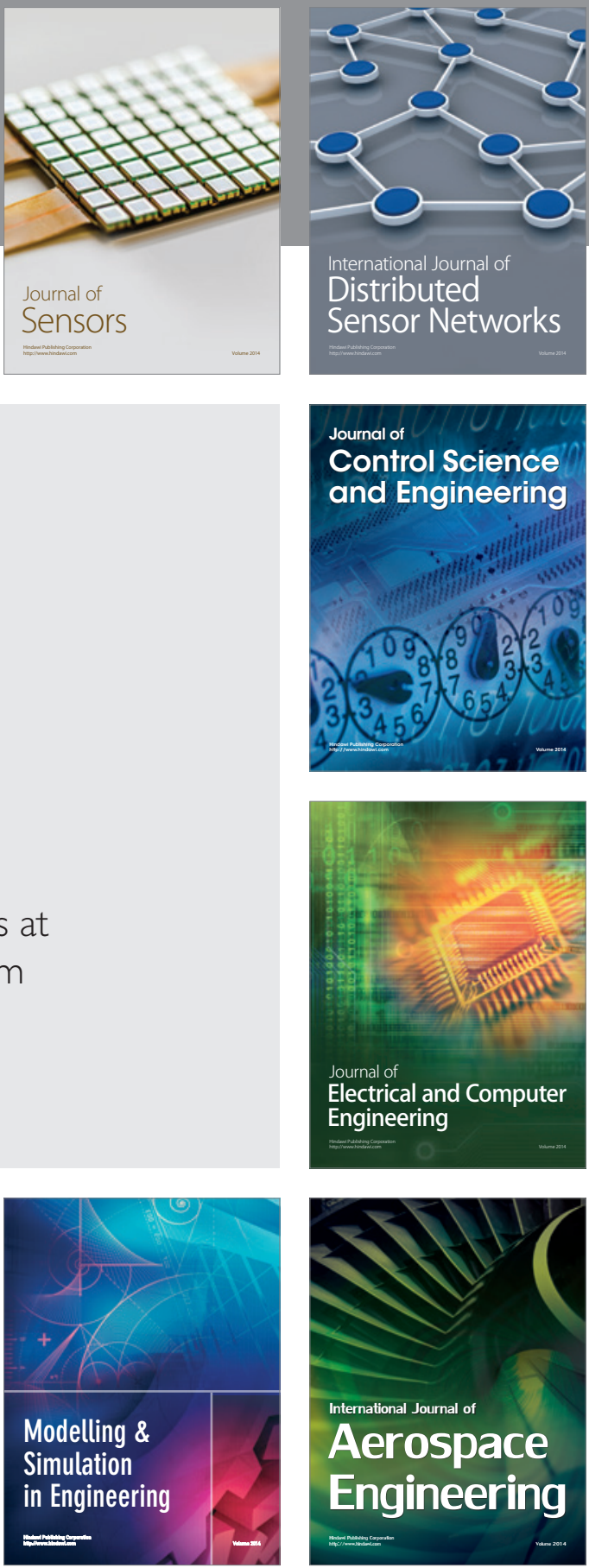

Journal of

Control Science

and Engineering
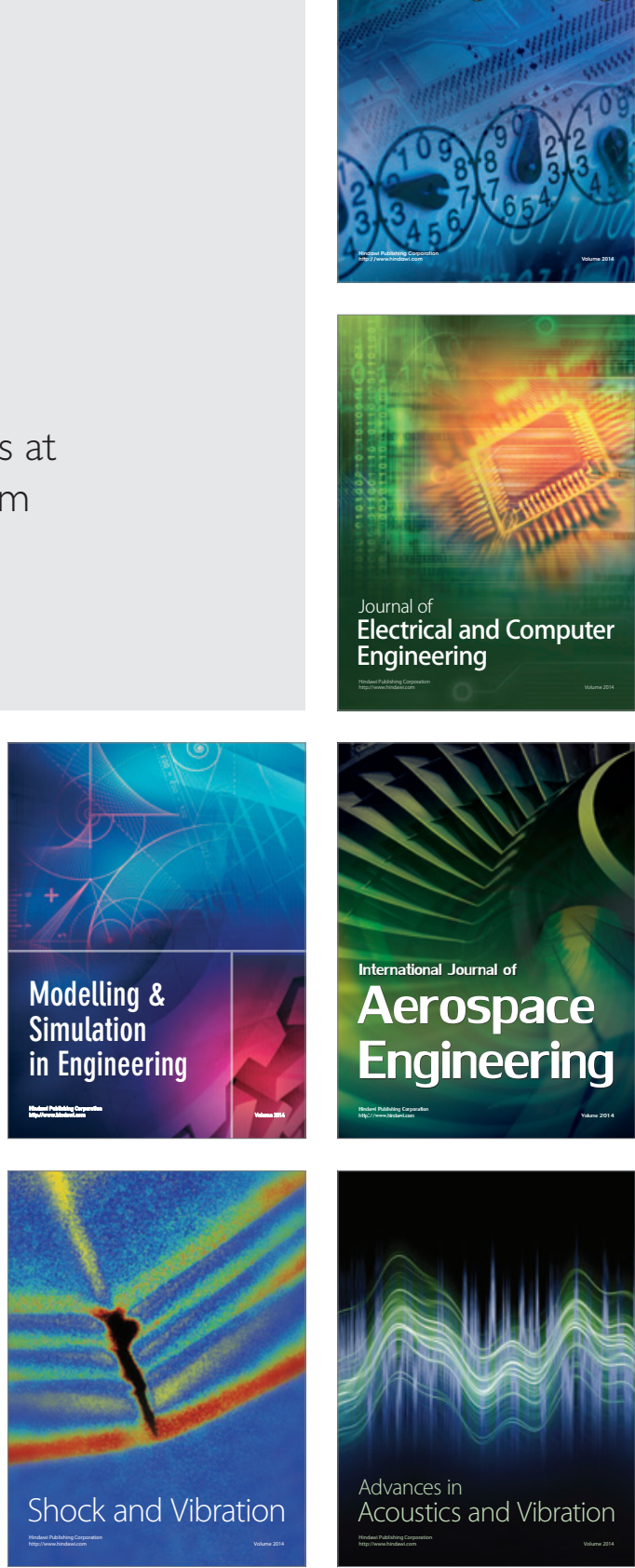